\title{
Subspaces intersecting each element of a regulus in one point, André-Bruck-Bose representation and clubs
}

\author{
Michel Lavrauw* Corrado Zanella ${ }^{\dagger}$ \\ Dipartimento di Tecnica e Gestione dei Sistemi Industriali \\ Università di Padova \\ Stradella S. Nicola 3 \\ 36100 Vicenza, Italy \\ \{michel.lavrauw, corrado.zanella\}@unipd.it
}

Submitted: Sep 3, 2014; Accepted: Feb 10, 2016; Published: Feb 19, 2016

Mathematics Subject Classifications: 51E20

\begin{abstract}
In this paper results are proved with applications to the orbits of $(n-1)$ dimensional subspaces disjoint from a regulus $\mathcal{R}$ of $(n-1)$-subspaces in $\mathrm{PG}(2 n-1, q)$, with respect to the subgroup of $\operatorname{PGL}(2 n, q)$ fixing $\mathcal{R}$. Such results have consequences on several aspects of finite geometry. First of all, a necessary condition for an $(n-1)$ subspace $U$ and a regulus $\mathcal{R}$ of $(n-1)$-subspaces to be extendable to a Desarguesian spread is given. The description also allows to improve results in [2] on the AndréBruck-Bose representation of a $q$-subline in $\mathrm{PG}\left(2, q^{n}\right)$. Furthermore, the results in this paper are applied to the classification of linear sets, in particular clubs.
\end{abstract}

Keywords: club; linear set; subplane; André-Bruck-Bose representation; Segre variety

\section{Introduction}

The $(n-1)$-dimensional projective projective space over the field $F$ is denoted by $\mathrm{PG}(n-$ $1, F)$ or $\mathrm{PG}(n-1, q)$ if $F$ is the finite field of order $q$ (denoted by $\mathbb{F}_{q}$ ). The set of nonzero elements of a field $F$ will be denoted by $F^{*}$, and similarly, the set of nonzero vectors of a vector space $V$ by $V^{*}$. If $L$ is an extension field $\mathbb{F}_{q}$, then the projective space defined by

${ }^{*}$ The research of this author is supported by the Research Foundation Flanders-Belgium (FWOVlaanderen) and by a Progetto di Ateneo from Università di Padova (CPDA113797/11).

${ }^{\dagger}$ The research of this author is supported by the Italian Ministry of Education, University and Research (PRIN 2012 project "Strutture geometriche, combinatoria e loro applicazioni"). 
the $\mathbb{F}_{q^{-}}$-vector space induced by $L^{d}$ is also denoted by $\mathrm{PG}_{q}\left(L^{d}\right)$. For a (sets of) subspace(s) $R$ of a vector space or a projective space, the notation $\langle R\rangle$ is used to denote the subspace generated by (the elements of) $R$. In case there is any ambiguity about the coefficient field, then the notation $\langle R\rangle_{q}$ will be used, to denote that the considered subspace is the one generated over $\mathbb{F}_{q}$. In this case the terminology of $\mathbb{F}_{q}$-span will sometimes be used. For example, if $S$ is a set of two points on the projective line $\operatorname{PG}\left(1, q^{2}\right)$, then $\langle S\rangle_{q}$ denotes the $\mathbb{F}_{q^{-}}$-subline defined by $S$, while $\langle S\rangle_{q^{2}}$ coincides with the whole projective line PG $\left(1, q^{2}\right)$.

For further notation and general definitions employed in this paper the reader is referred to $[9,11,13]$.

For more information on Desarguesian spreads see [1].

This paper is structured as follows. In Section 2 subspaces which intersect each element of a regulus in one point are studied and a result from [4] is generalised. Section 3 contains one of the main results of this paper, determining the order of the normal rational curves obtained from $n$-dimensional subspaces on an external $(n-1)$-dimensional subspace with respect to a regulus in $\mathrm{PG}(2 n-1, q)$, obtained from a point and a subline after applying the field reduction map to $\mathrm{PG}\left(1, q^{n}\right)$. This leads to a necessary condition on the existence of a Desarguesian spread containing a subspace and regulus (Corollary 7). The André-BruckBose representation of sublines and subplanes of a finite projective plane is studied ${ }^{1}$ in Section 4 and improvements are obtained with respect to the known results $[3,14,16,2]$. The results from the first sections of this paper are then applied to the classification problem for clubs of rank three in $\mathrm{PG}\left(1, q^{n}\right)$ in Section 5. A study of the incidence structure of the clubs in $\mathrm{PG}\left(1, q^{n}\right)$ after field reduction yields to a partial classification, concluding that the orbits of clubs under $\operatorname{PGL}\left(2, q^{n}\right)$ are at least $k-1$, where $k$ stands for the number of divisors of $n$. The paper concludes with an appendix discussing a result motivated by Burau [4] for the complex numbers: the result is extended to general algebraically closed fields; a new proof is provided; and counterexamples are given to some of the arguments used in the original proof.

\section{Subspaces intersecting each element of a regulus in one point}

Let $\mathcal{R}$ be a regulus of subspaces in a projective space and let $S$ be any subspace of $\langle\mathcal{R}\rangle$. Questions about the properties of the set of intersection points, which for reasons of simplicity of notation we will denote by $S \cap \mathcal{R}$, often turn up while investigating objects in finite geometry. If $S$ intersects each element of the regulus $\mathcal{R}$ in a point, then the intersection $S \cap \mathcal{R}$ is a normal rational curve, see Lemma 1 . This was already pointed out in $[4$, p.173] with a proof originally intended for complex projective spaces, but actually holding in a more general setting. The notation of [4] will be partly adopted.

The Segre variety representing the Cartesian product $\mathrm{PG}(n, F) \times \mathrm{PG}(m, F)$ in $\mathrm{PG}((n+$ $1)(m+1)-1, F)$ is denoted by $\mathcal{S}_{n, m, F}$. It is well known that $\mathcal{S}_{n, m, F}$ contains two families $\mathcal{S}_{n, m, F}^{I}$ and $\mathcal{S}_{n, m, F}^{I I}$ of maximal subspaces of dimensions $n$ and $m$, respectively. When

\footnotetext{
${ }^{1} \mathrm{~A}$ different study of $\mathbb{F}_{q^{k}}$-sublines and $\mathbb{F}_{q^{k}}$-subplanes of $\mathrm{PG}\left(2, q^{n}\right)$ in this representation can be found in [15].
} 
convenient, the notation $S^{I}$ or $S^{I I}$ will be used for a subspace belonging to the first or second family. The points of $\mathcal{S}_{n, m, F}$ may be represented as one-dimensional subspaces spanned by rank one $(m+1) \times(n+1)$ matrices. This is the standard example of a regular embedding of product spaces, see [17]. Note that in the finite case it is possible to embed product spaces in projective spaces of smaller dimension (see e.g. [7]). A regulus $\mathcal{R}$ of $(n-1)$-dimensional subspaces can also be defined as $\mathcal{S}_{n-1,1, F}^{I}$.

Lemma 1. Let $n>1$ be an integer, and $F$ a field. Let $S_{t}$ be a t-subspace of $\mathrm{PG}(2 n-1, F)$ intersecting each $S^{I} \in \mathcal{S}_{n-1,1, F}^{I}$ in precisely one point. Define $\Phi=S_{t} \cap \mathcal{S}_{n-1,1, F}$, and assume $\langle\Phi\rangle=S_{t}$. Then $|F| \geqslant t$ and the following properties hold.

(i) The set $\Phi$ is a normal rational curve of order $t$.

(ii) Let $\Xi^{I} \in \mathcal{S}_{n-1,1, F}^{I}$. Then the set $S\left(\Phi, \Xi^{I}\right)$ of the intersections of $\Xi^{I}$ with all transversal lines $l^{I I}$ such that $l^{I I} \cap \Phi \neq \emptyset$ is a normal rational curve of order $t$ or $t-1$ if $|F|=t$, and of order $t-1$ if $|F|>t$.

(iii) If $\Phi$ is contained in a subvariety $\mathcal{S}_{t-1,1, F}$ of $\mathcal{S}_{n-1,1, F}$, then homogeneous coordinates can be chosen such that $\Phi$ is represented parametrically by

$$
\left\langle\left(\begin{array}{cccc}
y_{0}^{t} & y_{0}^{t-1} y_{1} & \ldots & y_{0} y_{1}^{t-1} \\
y_{0}^{t-1} y_{1} & y_{0}^{t-2} y_{1}^{2} & \ldots & y_{1}^{t}
\end{array}\right)\right\rangle, \quad\left(y_{0}, y_{1}\right) \in\left(F^{2}\right)^{*}
$$

and $S\left(\Phi, \Xi^{I}\right)$, for $z_{0}, z_{1}$ depending only on $\Xi^{I}$, by

$$
\left\langle\left(\begin{array}{llll}
y_{0}^{t-1} z_{0} & y_{0}^{t-2} y_{1} z_{0} & \ldots & y_{1}^{t-1} z_{0} \\
y_{0}^{t-1} z_{1} & y_{0}^{t-2} y_{1} z_{1} & \ldots & y_{1}^{t-1} z_{1}
\end{array}\right)\right\rangle, \quad\left(y_{0}, y_{1}\right) \in\left(F^{2}\right)^{*}
$$

Proof. (i), (iii) The proof in [4, Sect.41 no.3], which is offered for $F=\mathbb{C}$, works exactly the same provided that $|F|>t$ or, more generally, that $\Phi$ is contained in some subvariety $\mathcal{S}_{t-1,1, F}$ of $\mathcal{S}_{n-1,1, F}$. In case $|F| \leqslant t$, the size of $\Phi$ being $|F|+1$ implies $|F|=t$, so $\Phi$ is just a set of $t+1$ independent points in a subspace isomorphic to $\mathrm{PG}(t, t)$, hence $\Phi$ is a normal rational curve of order $t$.

(ii) The case $|F|>t$ is proved in [4] immediately after the corollary at p. 175. If $|F| \leqslant$ $t$, then $|F|=t$ and two cases are possible. If $\Phi$ is contained in some $\mathcal{S}_{t-1,1, F} \subseteq \mathcal{S}_{n-1,1, F}$, Burau's proof is still valid as was mentioned in case (ii); so, $S\left(\Phi, \Xi^{I}\right)$ is a normal rational curve of order $t-1=|F|-1$. Otherwise $S\left(\Phi, \Xi^{I}\right)$ is an independent $(t+1)$-set, hence a normal rational curve of order $|F|$.

Remark 2. If $|F|=t$ both cases in Lemma 1 (ii) can occur. The following two examples use the Segre embedding $\sigma=\sigma_{t-1,1, F}$ of the product space $\mathrm{PG}(t-1, t) \times \mathrm{PG}(1, t)$ in $\mathrm{PG}(2 t-$ $1, t)$. Let $\left\{s_{0}, s_{1}, \ldots, s_{t}\right\}$ be the set of points on $\mathrm{PG}(1, t)$ and suppose $\left\{r_{0}, r_{1}, \ldots, r_{t}\right\}$ is a set of $t+1$ points in $\mathrm{PG}(t-1, t)$. Put $\Xi^{I}=\sigma\left(\mathrm{PG}(1, t) \times s_{0}\right)$ and $\Phi:=\left\{\sigma\left(r_{i} \times s_{i}\right): i=\right.$ $0,1, \ldots, t\}$. Then $\Phi$ consists of $t+1$ points on the Segre variety $\mathcal{S}_{t-1,1, F}$. Depending on the set $\left\{r_{0}, r_{1}, \ldots, r_{t}\right\}$ one obtains the two cases described in Lemma 1 (ii). 
a. If $\left\{r_{0}, r_{1}, \ldots, r_{t}\right\}$ is a frame of a hyperplane of $\mathrm{PG}(t-1, t)$ then $\Phi$ generates a $t$ dimensional subspace of $\mathrm{PG}(2 t-1, t)$ intersecting $\mathcal{S}_{t-1,1, F}$ in $\Phi$ and $S\left(\Phi, \Xi^{I}\right)$ is a normal rational curve of order $t-1$.

b. If $\left\{r_{0}, r_{1}, \ldots, r_{t}\right\}$ generates $\mathrm{PG}(t-1, t)$ then $\Phi$ generates a $t$-dimensional subspace of $\mathrm{PG}(2 t-1, t)$ intersecting $\mathcal{S}_{t-1,1, F}$ in $\Phi$ and $S\left(\Phi, \Xi^{I}\right)$ is a normal rational curve of order $t$.

Remark 3. By (1) and (2), the map $\alpha: \Phi \rightarrow S\left(\Phi, \Xi^{I}\right)$ defined by the condition that $X$ and $X^{\alpha}$ are on a common line in $\mathcal{S}_{n-1,1, F}^{I I}$ is related to a projectivity between the parametrizing projective lines. Such an $\alpha$ is also called a projectivity.

\section{The order of normal rational curves contained in $\mathcal{S}_{n-1,1, q}$}

Here $n \geqslant 2$ is an integer. The field reduction map $\mathcal{F}_{m, n, q}$ from $\mathrm{PG}\left(m-1, q^{n}\right)$ to $\mathrm{PG}(m n-$ $1, q)$ will also be denoted by $\mathcal{F}$. If $S$ is a set of points, in $\mathrm{PG}\left(m-1, q^{n}\right)$, then $\mathcal{F}(S)$ is a set of subspaces, whose union, as a set of points will be denoted by $\tilde{\mathcal{F}}(S)$. The $\mathbb{F}_{q^{h} \text {-span }}$ of a subset $b$ of $\operatorname{PG}\left(d, q^{n}\right)$ is denoted by $\langle b\rangle_{q^{h}}$.

Proposition 4. Let $b$ be a q-subline of $\mathrm{PG}\left(1, q^{n}\right)$, and let $\Theta$ be a point of $\mathrm{PG}\left(1, q^{n}\right)$. Let $(1, \zeta)$ and $\left(1, \zeta^{\prime}\right)$ be homogeneous coordinates of $\Theta$ with respect to two reference frames for $\langle b\rangle_{q^{n}}$, each of which consists of three points of $b$. Then $\mathbb{F}_{q}(\zeta)=\mathbb{F}_{q}\left(\zeta^{\prime}\right)$.

Proof. Homogeneous coordinates of a point in both reference frames, say $\left(x_{0}, x_{1}\right)$ and $\left(x_{0}^{\prime}, x_{1}^{\prime}\right)$, are related by an equation of the form $\rho\left(x_{0}^{\prime} x_{1}^{\prime}\right)^{T}=A\left(x_{0} x_{1}\right)^{T}, \rho \in \mathbb{F}_{q^{n}}^{*}, A \in$ $\operatorname{GL}(2, q)$. Hence $\left(\rho \rho \zeta^{\prime}\right)^{T}=A(1 \zeta)^{T}$ and this implies $\zeta^{\prime} \in \mathbb{F}_{q}(\zeta)$. The proof of $\zeta \in \mathbb{F}_{q}\left(\zeta^{\prime}\right)$ is similar.

By Proposition 4 , given a $q$-subline $b$ in a finite projective space $\operatorname{PG}\left(d, q^{n}\right)$ and a point $\Theta \in\langle b\rangle_{q^{n}}$, with homogeneous coordinates $(1, \zeta)$ with respect to a reference frame of $\langle b\rangle_{q^{n}}$ consisting of three points of $b$, the degree of $\Theta$ over $b$, denoted by $[\Theta: b]$, is well-defined in terms of the field extension degree as follows: $[\Theta: b]=\left[\mathbb{F}_{q}(\zeta): \mathbb{F}_{q}\right]$.

This $[\Theta: b]$ also equals the minimum integer $m$ such that a subgeometry $\Sigma \cong \operatorname{PG}\left(d, q^{m}\right)$ exists containing both $b$ and $\Theta$.

Proposition 5. Any n-subspace of $\mathrm{PG}(2 n-1, q)$ containing an $(n-1)$-subspace $S^{I} \in$ $\mathcal{S}_{n-1,1, q}^{I}$ intersects $\mathcal{S}_{n-1,1, q}$ in the union of $S^{I}$ and a line in $\mathcal{S}_{n-1,1, q}^{I I}$.

Theorem 6. Let $b$ be a q-subline of $\mathrm{PG}\left(1, q^{n}\right)$, and $\Theta \notin b$ a point of $\mathrm{PG}\left(1, q^{n}\right)$. Then in $\mathrm{PG}(2 n-1, q)$ any $n$-subspace $\mathcal{H}$ containing $\mathcal{F}(\Theta)$ intersects the Segre variety $\mathcal{S}_{n-1,1, q}=$ $\tilde{\mathcal{F}}(b)$, in a normal rational curve whose order is $\min \{q,[\Theta: b]\}$.

Proof. Set $L=\mathbb{F}_{q^{n}}, F=\mathbb{F}_{q}$. Without loss of generality, $\operatorname{PG}(2 n-1, q)=\mathrm{PG}_{q}\left(L^{2}\right)$, $\mathcal{F}(b)=\left\{L(x, y) \mid(x, y) \in\left(F^{2}\right)^{*}\right\}^{2}$, and $\Theta=L(1, \xi)$ with $[F(\xi): F]=[\Theta: b]$. The

\footnotetext{
${ }^{2}$ For $x, y \in L, F(x, y)=\langle(x, y)\rangle_{q}$, and $L(x, y)=\langle(x, y)\rangle_{q^{n}}$.
} 
$n$-subspace $\mathcal{H}$ intersects $L(1,0)$ in one point $Y$ of the form $Y=F(\theta, 0), \theta \in L^{*}$. For any $x \in F$, seeking for the intersection $\langle\mathcal{F}(\Theta), Y\rangle_{q} \cap L(x, 1)$, or

$$
\langle L(1, \xi), F(\theta, 0)\rangle_{q} \cap L(x, 1)
$$

gives two equations in $\alpha, \beta \in L$ :

$$
\alpha+\theta=\beta x, \quad \alpha \xi=\beta,
$$

whence $\beta=\theta\left(x-\xi^{-1}\right)^{-1}$. The intersection point is then $F\left(x \theta\left(x-\xi^{-1}\right)^{-1}, \theta\left(x-\xi^{-1}\right)^{-1}\right)$. So, for $\Xi=L(0,1)$, the set of the intersections of $\Xi$ with all lines in $\mathcal{S}_{n-1,1, q}^{I I}$ which meet $\mathcal{H}$ is

$$
S\left(\mathcal{H} \cap \mathcal{S}_{n-1,1, q}, \Xi\right)=\left\{F\left(0, \theta\left(x-\xi^{-1}\right)^{-1}\right) \mid x \in \mathcal{F}_{q}\right\} \cup\{F(0, \theta)\} .
$$

This $S\left(\mathcal{H} \cap \mathcal{S}_{n-1,1, q}, \Xi\right)$ is obtained by inversion from the line joining the points $F\left(0, \theta^{-1}\right)$ and $F\left(0, \theta^{-1} \xi^{-1}\right)$. By [10, Theorem 5], $\mathcal{C}_{Y}$ is a normal rational curve of order $\delta^{\prime}=$ $\min \left\{q,\left[F\left(\xi^{-1}\right): F\right]-1\right\}=\min \{q,[\Theta: b]-1\}$. Now apply lemma 1 for $S_{t}=\left\langle\mathcal{H} \cap \mathcal{S}_{n-1,1, q}\right\rangle_{q}:$ if $t \geqslant q$, then $t=q$ and $\delta^{\prime}=q$ or $\delta^{\prime}=q-1$, so $[\Theta: b] \geqslant q$ and $t=\min \{q,[\Theta: b]\}$. If on the contrary $t<q$, then $t-1=\delta^{\prime}=[\Theta: b]-1$, so $t=[\Theta: b]$ and $t=\min \{q,[\Theta: b]\}$ again.

An important consequence of the above result answers the question of the existence of a Desarguesian spread containing a given regulus $\mathcal{R}$ and a subspace disjoint from $\mathcal{R}$.

Corollary 7. If a regulus $\mathcal{R}=\mathcal{S}_{n-1,1, q}$ and an $(n-1)$-dimensional subspace $U$, disjoint from $\mathcal{R}$, in $\mathrm{PG}(2 n-1, q)$ are contained in a Desarguesian spread then there is an integer $c$ such that any $n$-subspace $\mathcal{H}$ containing $U$ intersects $\mathcal{R}$ in a normal rational curve of order $c$.

The following remark illustrates that this necessary condition is not always satisfied.

Remark 8. For $n>2$ by using the package FinInG [5] of GAP [6] examples can be given of $(n-1)$-subspaces disjoint from $\mathcal{S}_{n-1,1, q}$ contained in $n$-subspaces intersecting the Segre variety in normal rational curves of distinct orders. We include one explicit example. Let $q=4, \mathbb{F}_{q}=\mathbb{F}_{2}(\omega)$, with $\omega^{2}+\omega+1=0$. Let $\mathcal{R}$ be the regulus of 3-dimensional subspaces of $\operatorname{PG}(7,4)$ obtained from the standard subline $\mathrm{PG}(1, q)$ in $\mathrm{PG}\left(1, q^{4}\right)$, and put

$$
\begin{array}{r}
S_{3}:=\left\langle\left(1,0,0,0, \omega^{2}, 1,0,1\right),\left(0,1,0,0,1, \omega^{2}, 0, \omega^{2}\right),\right. \\
\left.(0,0,1,0,0, \omega, 1, \omega),\left(0,0,0,1, \omega^{2}, \omega^{2}, \omega, 1\right)\right\rangle .
\end{array}
$$

Then $S_{3}$ is a three-dimensional subspace disjoint from the regulus $\mathcal{R}$. Moreover, the 4dimensional subspace $\left\langle S_{3},(1,0,0,0,0,0,0,0)\right\rangle$ intersects the regulus $\mathcal{R}$ in a normal rational curve of order 4 , while the 4-dimensional subspace $\left\langle S_{3},\left(0,1,0, \omega^{2}, 0,0,0,0\right)\right\rangle$ intersects $\mathcal{R}$ in a conic. 


\section{André-Bruck-Bose representation}

The André-Bruck-Bose representation of a Desarguesian affine plane of order $q^{n}$ is related to the image of $\operatorname{PG}\left(2, q^{n}\right)$, under the field reduction map $\mathcal{F}$, by means of the following straightforward result.

Proposition 9. Let $\mathcal{D}$ be the Desarguesian spread in $\mathrm{PG}(3 n-1, q)$ obtained after applying the field reduction map $\mathcal{F}$ to the set of points of $\mathrm{PG}\left(2, q^{n}\right), l_{\infty}$ a line in $\mathrm{PG}\left(2, q^{n}\right)$, and $\mathcal{K}$ a $(2 n)$-subspace of $\mathrm{PG}(3 n-1, q)$, containing the spread $\mathcal{F}\left(l_{\infty}\right)$. Take $\mathrm{PG}\left(2, q^{n}\right) \backslash l_{\infty}$ and $\mathcal{K} \backslash\left\langle\mathcal{F}\left(l_{\infty}\right)\right\rangle_{q}$ as representatives of $\mathrm{AG}\left(2, q^{n}\right)$ and $\mathrm{AG}(2 n, q)$, respectively. Then the map $\varphi: \operatorname{AG}\left(2, q^{n}\right) \rightarrow \operatorname{AG}(2 n, q)$ defined by $\varphi(X)=\mathcal{F}(X) \cap \mathcal{K}$ for any $X \in \operatorname{AG}\left(2, q^{n}\right)$ is a bijection, mapping lines of $\mathrm{AG}\left(2, q^{n}\right)$ into $n$-subspaces of $\operatorname{AG}(2 n, q)$ whose $(n-1)$ subspaces at infinity belong to the spread $\mathcal{F}\left(l_{\infty}\right)$.

The notation in Proposition 9 is assumed to hold in the whole section. The following result improves [2, Theorems 3.3 and 3.5], by determining the order of the involved normal rational curves.

Theorem 10. Let $b$ be a q-subline of $\mathrm{PG}\left(2, q^{n}\right)$, not contained in $l_{\infty}$. Set $\Theta=\langle b\rangle_{q^{n}} \cap l_{\infty}$. Then the André-Bruck-Bose representation $\varphi\left(b \backslash l_{\infty}\right)$ is the affine part of a normal rational curve whose order is $\delta=\min \{q,[\Theta: b]\}$. More precisely, if $\delta=1$, then $\varphi\left(b \backslash l_{\infty}\right)$ is an affine line; if $\delta>1$, then $b \cap l_{\infty}=\emptyset$, and $\varphi(b)$ is a normal rational curve with no points at infinity.

Proof. The intersection $\mathcal{H}=\langle\mathcal{F}(b)\rangle_{q} \cap \mathcal{K}$ is an $n$-space containing $\mathcal{F}(\Theta)$, and contained in the span of the Segre variety $\mathcal{S}_{n-1,1, q}=\tilde{\mathcal{F}}(b)$, as defined at the start of Section 3. The result follows from Proposition 5 and Theorem 6.

The results in [2, Theorems 3.3 and 3.5] also characterize the normal rational curves arising from $q$-sublines in $\mathrm{AG}\left(2, q^{n}\right)$.

In $[3,14,16]$ for $n=2$ and $[2$, Theorem 3.6 (a)(b)] for any $n$ the André-Bruck-Bose representation of a $q$-subplane tangent to a line at the infinity is described. Further properties are stated in the following theorem:

Theorem 11. Let $B$ be a q-subplane of $\mathrm{PG}\left(2, q^{n}\right)$ that is tangent to $l_{\infty}$ at the point $T$. Let $b$ be a line of $B$ not through $T, \Theta=\langle b\rangle_{q^{n}} \cap l_{\infty}$, and $\delta=\min \{q,[\Theta: b]\}$. Then there are a normal rational curve $\mathcal{C}_{0}$ of order $\delta$ in the $n$-subspace $\varphi\left(\langle b\rangle_{q^{n}}\right)$, a normal rational curve $\mathcal{C}_{1} \subset \mathcal{F}(T)$ of order $\delta^{\prime}$, with

$$
\delta^{\prime}\left\{\begin{array}{l}
=[\Theta: b]-1 \quad \text { for } q>[\Theta: b] \\
\in\{q-1, q\} \quad \text { otherwise }
\end{array}\right.
$$

and a projectivity $\kappa: \mathcal{C}_{0} \rightarrow \mathcal{C}_{1}$ (in the sense of Remark 3), such that $\varphi\left(B \backslash l_{\infty}\right)$ is the ruled surface union of all lines $X X^{\kappa}$ for $X \in \mathcal{C}_{0}$. 
Proof. By Theorem $10, \mathcal{C}_{0}:=\varphi(b)$ is a normal rational curve of order $\delta$ in the $n$-subspace $\varphi\left(\langle b\rangle_{q^{n}} \backslash l_{\infty}\right)$, and for any $P=\varphi(X) \in \mathcal{C}_{0}$, the subline $T X$ of $B$ corresponds to an affine line $P P^{\kappa}$ with $P^{\kappa} \in \mathcal{F}(T)$ at infinity. Define $\mathcal{C}_{1}=\left\{P^{\kappa} \mid P \in \mathcal{C}_{0}\right\}$.

By the field reduction map $\mathcal{F}=\mathcal{F}_{3, n, q}$, the subplane $B$ is mapped to $\mathcal{F}(B)$ which is the set of all maximal subspaces of the first family in $\mathcal{S}_{n-1,2, q} \subset \operatorname{PG}(3 n-1, q)$. Considering $\mathbb{F}_{q^{n}}$ as an $\mathbb{F}_{q^{-} \text {-vector space, the homomorphism }}$

$$
\mathbb{F}_{q^{n}} \times \mathbb{F}_{q}^{3} \rightarrow \mathbb{F}_{q^{n}} \otimes \mathbb{F}_{q}^{3}:(\lambda, v) \mapsto \lambda \otimes v
$$

corresponds to a projective embedding $g: \operatorname{PG}(n-1, q) \times B \rightarrow \mathcal{S}_{n-1,2, q}$ whose image is $\mathcal{S}_{n-1,2, q}$, and such that $\mathcal{F}(X)=(\operatorname{PG}(n-1, q) \times X)^{g}$ for any point $X$ in $B$. It holds $\varphi\left(B \backslash l_{\infty}\right)=\mathcal{S}_{n-1,2, q} \cap \mathcal{K} \backslash \mathcal{F}(T)$. For any point $U$ in $B$ define

$$
\kappa_{U}:(X, Y)^{g} \in \mathcal{S}_{n-1,2, q} \mapsto(X, U)^{g} \in \mathcal{F}(U) .
$$

Note that for any $Y \in B$, the restriction of $\kappa_{U}$ to $\mathcal{F}(Y)$ is a projectivity. For any $U \in b$, using the notation from Lemma 1 it holds $\mathcal{C}_{0}^{\kappa_{U}}=S\left(\mathcal{C}_{0}, \mathcal{F}(U)\right)$, and as a consequence, $\mathcal{C}_{0}^{\kappa_{U}}$ is a normal rational curve of order $\delta^{\prime}$ as in (3). Now, since for any $P \in \mathcal{C}_{0}$, say $P=\left(X_{P}, Y_{P}\right)^{g}$, the points $P, P^{\kappa}$ and $P^{\kappa_{T}}$ are on the plane $\left(X_{P} \times B\right)^{g} \in \mathcal{S}_{n-1,2, q}^{I I}$, and $P^{\kappa}, P^{\kappa_{T}} \in \mathcal{F}(T)$, it follows that $P^{\kappa}=P^{\kappa_{T}}$. It also follows that $\mathcal{C}_{1}=\mathcal{C}_{0}^{\kappa_{U} \kappa_{T}}=S\left(\mathcal{C}_{0}, \mathcal{F}(U)\right)^{\kappa_{T}}$, and hence $\mathcal{C}_{1}$ is a normal rational curve of order $\delta^{\prime}$ as in (3). Finally, $\kappa_{U}: \mathcal{C}_{0} \rightarrow S\left(\mathcal{C}_{0}, \mathcal{F}(U)\right)$ is a projectivity as defined in Remark 3 , and hence so is $\kappa$.

\section{On the classification of clubs}

An $\mathbb{F}_{q}$-club (or simply a club) in $\mathrm{PG}\left(1, q^{n}\right)$ is an $\mathbb{F}_{q}$-linear set of rank three, having a point of weight two, called the head of the club. An $\mathbb{F}_{q}$-club has $q^{2}+1$ points, and the non-head points have weight one. From now on it will be assumed that $n>2$. The next proposition is a straightforward consequence of the representation of linear sets as projections of subgeometries [12, Theorem 2].

Proposition 12. Let $L$ be an $\mathbb{F}_{q^{-}}$club in $\mathrm{PG}\left(1, q^{n}\right) \subset \mathrm{PG}\left(2, q^{n}\right)$. Then there are a $q$ subplane $\Sigma$ of $\mathrm{PG}\left(2, q^{n}\right)$, a q-subline $b$ in $\Sigma$, and a point $\Theta \in\langle b\rangle_{q^{n}} \backslash b$, such that $L$ is the projection of $\Sigma$ from the center $\Theta$ onto the axis $\mathrm{PG}\left(1, q^{n}\right)$.

As before the notation $\mathcal{F}$ and $\tilde{\mathcal{F}}$ is used, where $\mathcal{F}=\mathcal{F}_{2, n, q}$ denotes the field reduction map from $\mathrm{PG}\left(1, q^{n}\right)$ to $\mathrm{PG}(2 n-1, q)$.

Proposition 13. Let $L$ be an $\mathbb{F}_{q}$ club of $\mathrm{PG}\left(1, q^{n}\right)$ with head $\Upsilon$. Then $\tilde{\mathcal{F}}(L)$ contains two collections of subspaces, say $F_{1}$ and $F_{2}$, satisfying the following properties.

(i) The subspaces in $F_{1}$ are $(n-1)$-dimensional, are pairwise disjoint, and any subspace in $F_{1}$ is disjoint from $\mathcal{F}(\Upsilon)$.

(ii) Any subspace in $F_{2}$ is a plane and intersects $\mathcal{F}(\Upsilon)$ in precisely a line. 
(iii) Any point of $\mathcal{F}(\Upsilon)$ belongs to exactly $q+1$ planes in $F_{2}$.

(iv) If $L$ is not isomorphic to $\mathrm{PG}\left(1, q^{2}\right)$, and $l$ is any line of $\mathrm{PG}(2 n-1, q)$ contained in $\tilde{\mathcal{F}}(L)$, then $l$ is contained in $\mathcal{F}(\Upsilon)$ or in a subspace in $F_{1} \cup F_{2}$.

Proof. The assumptions imply the existence of $\Sigma$ and a $q$-subline $b$ in $\Sigma$ as in Proposition 12. The assertions are a consequence of the fact that $\tilde{\mathcal{F}}(\Sigma)$ is a Segre variety $\mathcal{S}_{n-1,2, q}$ in $\mathrm{PG}(3 n-1, q)$. Let

$$
p_{1}: \operatorname{PG}\left(2, q^{n}\right) \backslash \Theta \rightarrow \operatorname{PG}\left(1, q^{n}\right)
$$

be the projection with center $\Theta$, associated with

$$
p_{2}: \mathrm{PG}(3 n-1, q) \backslash \mathcal{F}(\Theta) \rightarrow \mathrm{PG}(2 n-1, q) .
$$

The collections $F_{1}$ and $F_{2}$ are defined as follows:

$$
F_{1}=\left\{\mathcal{F}\left(p_{1}(X)\right) \mid X \in \Sigma \backslash b\right\}=\mathcal{F}(L) \backslash \mathcal{F}(\Upsilon), \quad F_{2}=\left\{p_{2}\left(V^{I I}\right) \mid V^{I I} \in \tilde{\mathcal{F}}(\Sigma)^{I I}\right\}
$$

The assertion (i) is straightforward, as well as $\operatorname{dim}(V)=2$ for any $V \in F_{2}$. For any $V^{I I} \in$ $\tilde{\mathcal{F}}(\Sigma)^{I I}$, the intersection $V^{I I} \cap\langle\tilde{\mathcal{F}}(b)\rangle_{q}$ is a line, and this with $p_{2}^{-1}(\mathcal{F}(\Upsilon))=\langle\tilde{\mathcal{F}}(b)\rangle_{q} \backslash \mathcal{F}(\Theta)$ implies the second assertion in (ii). Next, let $P$ be a point in $\mathcal{F}(\Upsilon)$. A plane $V=p_{2}\left(V^{I I}\right)$ contains $P$ if, and only if, $V^{I I}$ intersects the $n$-subspace $\langle\mathcal{F}(\Theta), P\rangle_{q}$, that is, $V^{I I}$ intersects the normal rational curve $\mathcal{S}_{n-1,2, q} \cap\langle\mathcal{F}(\Theta), P\rangle_{q}$; this implies (iii).

Assume that a line $l \subset \tilde{\mathcal{F}}(L)$ exists which is neither contained in $\mathcal{F}(\Upsilon)$, nor in a $T \in F_{1} \cup F_{2}$. Let $Q$ be a point in $l \backslash \mathcal{F}(\Upsilon)$, and let $V \in F_{2}$ such that $Q \in V$. It holds $L=\mathcal{B}(V)$. Then $\mathcal{B}(l)$ is a $q$-subline of $L$. Suppose that a line $l^{\prime}$ in $V$ exists such that $\mathcal{B}\left(l^{\prime}\right)=\mathcal{B}(l)$. Since $\mathcal{B}(Q) \neq \mathcal{B}\left(Q^{\prime}\right)$ for any $Q^{\prime} \in V, Q^{\prime} \neq Q$, the line $l^{\prime}$ contains $Q$. Then $l, l^{\prime}$ are two distinct transversal lines in $\mathcal{B}(l)^{I I}$, a contradiction. Hence $\mathcal{B}\left(l^{\prime}\right) \neq \mathcal{B}(l)$ for any line $l^{\prime}$ in $V$, that is, $\mathcal{B}(l)$ is a so-called irregular subline [8]. By [8, Corollary 13], no irregular subline exists in $L$, and this contradiction implies (iv).

Proposition 14. Let $L$ be an $\mathbb{F}_{q}$-club with head $\Upsilon$. Let $\Theta$ be the point and $b$ be the subline as defined in Proposition 12. Then for any point $X$ in $\mathcal{F}(\Upsilon)$, the intersection lines of $\mathcal{F}(\Upsilon)$ with any $q$ distinct planes in $F_{2}$ containing $X$ span an s-dimensional subspace, where

(i) $s=[\Theta: b]-1$ if $q>[\Theta: b]$;

(ii) $s \in\{q-1, q\}$ if $q \leqslant[\Theta: b]$.

Proof. Let $p_{2}$ be the projection map as defined in the proof of Proposition 13, $X=p_{2}(P)$, and $\mathcal{H}=\langle\mathcal{F}(\Theta), P\rangle_{q}$. For any plane $V=p_{2}\left(V^{I I}\right)$, it holds $X \in V$ if, and only if $V^{I I} \cap \mathcal{H} \neq \emptyset$. The intersection $\mathcal{H} \cap \tilde{\mathcal{F}}(b)$ is a normal rational curve of order $\min \{q,[\Theta: b]\}$ (cf. Theorem 6). Let $V_{0}=p_{2}\left(V_{0}^{I I}\right)$ be the unique plane of $F_{2}$ through $X$ distinct from the $q$ planes chosen in the assumptions (cf. Proposition 13). Let $Q=\tilde{\mathcal{F}}(b) \cap V_{0}^{I I} ; \mathcal{B}(Q)$ is an $(n-1)$-subspace of $\tilde{\mathcal{F}}(b)^{I}$. Such $\mathcal{B}(Q)$ is mapped onto $\mathcal{B}(X)=\mathcal{F}(\Upsilon)$ by $p_{2}$. Assume $V_{i}=p_{2}\left(V_{i}^{I I}\right), i=1,2, \ldots, q$, are the $q$ planes chosen in the assumptions. Any $V_{i}^{I I}$, 
$i=1,2, \ldots, q$, intersects $\mathcal{H}$, hence $V_{i}^{I I} \cap \mathcal{B}(Q)$ is the intersection of $\mathcal{B}(Q)$ with a transversal line of $\tilde{\mathcal{F}}(b)$ intersecting the normal rational curve $\mathcal{H} \cap \tilde{\mathcal{F}}(b)$. By Lemma 1 (ii), the set

$$
S=\left\{V_{i}^{I I} \cap \mathcal{B}(Q) \mid i=1,2, \ldots, q\right\} \cup\{Q\}
$$

is a normal rational curve of order $s$ where $s$ takes the values as stated in $(i)$ and $(i i)$. Since $V_{i} \cap \mathcal{F}(\Upsilon)$ is the line through $X$ and a point of $p_{2}(S)$, distinct from $X$, the span of the intersection lines is the same as the span of $p_{2}(S)$.

Theorem 15. Let $\mathcal{I}_{n, q}$ be the set of integers $h$ dividing $n$ and such that $1<h<q$. For any $h \in \mathcal{I}_{n, q}$, let $L_{h}$ be the linear set obtained by projecting a q-subplane $\Sigma$ of $\mathrm{PG}\left(2, q^{n}\right)$ from a point $\Theta_{h}$ collinear with a q-subline $b$ in $\Sigma$ and such that $\left[\Theta_{h}: b\right]=h$. Then the set $\Lambda=\left\{L_{h} \mid h \in \mathcal{I}_{n, q}\right\}$ contains $\mathbb{F}_{q^{-}}$clubs in $\mathrm{PG}\left(1, q^{n}\right)$ all belonging to distinct orbits under $\operatorname{PGL}\left(2, q^{n}\right)$.

Proof. If $n$ is odd, then no club is isomorphic to $\mathrm{PG}\left(1, q^{2}\right)$. So, by Proposition 13 (iv), the families $F_{1}$ and $F_{2}$ are uniquely determined. The thesis is a consequence of Proposition 14 , taking into account that if $L$ and $L^{\prime}$ are projectively equivalent, then $\tilde{\mathcal{F}}(L)$ and $\tilde{\mathcal{F}}\left(L^{\prime}\right)$ are projectively equivalent in $\mathrm{PG}(2 n-1, q)$.

In order to deal with the case $n$ even, it is enough to show that in $\Lambda$ at most one club is isomorphic to $\mathrm{PG}\left(1, q^{2}\right)$. So assume $L_{h} \cong \mathrm{PG}\left(1, q^{2}\right)$. Then $\tilde{\mathcal{F}}\left(L_{h}\right)$ has a partition $\mathcal{P}_{1}$ in $(n-1)$-subspaces, and a partition $\mathcal{P}_{2}$ in 3-subspaces. From [8, Lemma 11] it can be deduced that any line contained in $\tilde{\mathcal{F}}\left(L_{h}\right)$ is contained in an element of $\mathcal{P}_{1}$ or $\mathcal{P}_{2}$. The intersections of a subspace $U$ of a family $\mathcal{P}_{i}$ with the elements of the other family form a line spread of $U$. Hence all planes in $F_{2}$ are contained in 3-subspaces of $\mathcal{P}_{2}$, and all planes of $F_{2}$ through a point $X$ in $\mathcal{F}(\Upsilon)$ meet $\mathcal{F}(\Upsilon)$ in the same line. By Proposition 14 this implies $h=2$.

\section{Acknowledgement}

The authors thank Hans Havlicek for his helpful remarks in the preparation of this paper.

\section{References}

[1] L. Bader, G. Lunardon: Desarguesian spreads. Ric. Mat. 60 (2011), 15-37.

[2] S.G. BARWICK, Wen-Ai JACKSON: Sublines and subplanes of PG $\left(2, q^{3}\right)$ in the Bruck-Bose representation in PG(6,q). Finite Fields App. 18 (2012), 93-107.

[3] R.C. Bose, J.W. Freeman, D.G. Glynn: On the intersection of two Baer subplanes in a finite projective plane. Utilitas Math. 17 (1980), 65-77.

[4] W. BurAu: Mehrdimensionale projektive und höhere Geometrie. VEB Deutscher Verlag der Wissenschaften, Berlin, 1961.

[5] FinInG - a GAP package - Finite Incidence Geometry, version 1.0, 2014. Bamberg, J.; Betten, A.; Cara, Ph.; De Beule, J.; Lavrauw, M. and Neunhöffer, M. 
[6] GAP - Groups, Algorithms, and Programming, Version 4.7.5; 2014. (http://www.gap-system.org)

[7] M. Lavrauw, J. Sheekey, C. Zanella: On embeddings of minimum dimension of $\mathrm{PG}(n, q) \times \operatorname{PG}(n, q)$. Des. Codes Cryptogr. 74 (2015), no. 2, 427-440.

[8] M. Lavrauw, G. Van de Voorde: On linear sets on a projective line. Des. Codes Cryptogr. 56 (2010), 89-104.

[9] M. Lavrauw, G. VAn De Voorde: Field reduction and linear sets in finite geometry. Topics in finite fields, 271-293, Contemp. Math., 632, Amer. Math. Soc., Providence, RI, 2015.

[10] M. Lavrauw, C. Zanella: Geometry of the inversion in a finite field and partitions of $\mathrm{PG}\left(2^{k}-1, q\right)$ in normal rational curves. J. Geom. 105 (2014), 103-110.

[11] M. Lavrauw, C. Zanella: Subgeometries and linear sets on a projective line. Finite Fields Appl. 34 (2015), 95-106.

[12] G. Lunardon, O. Polverino: Translation ovoids of orthogonal polar spaces. Forum Math. 16 (2004), 663-669.

[13] O. Polverino: Linear sets in finite projective spaces. Discrete Math. 310 (2010), 3096-3107.

[14] C.T. Quinn, L.R.A. CAsse: Concerning a characterisation of Buekenhout-Metz unitals, J. Geom. 52 (1995), 159-167.

[15] S. Rottey, J. Sheekey, G. Van de Voorde: Subgeometries in the André/Bruck-Bose representation. Finite Fields and Their Applications, 35 (2015) $115-138$.

[16] R. Vincenti: Alcuni tipi di varietà $\mathcal{V}_{2}$ di $S_{4, q}$ e sottopiani di Baer. Boll. Un. Mat. Ital. Suppl. (1980), no. 2, 31-44.

[17] C. Zanella: Universal properties of the Corrado Segre embedding. Bull. Belg. Math. Soc. Simon Stevin 3 (1996), 65-79.

\section{A Appendix: On a result in [4]}

In $[4$, p.175] the following result (Korollar) is stated for $F=\mathbb{C}$.

Corollary 16. Let $F$ be an algebraically closed field. If an s-subspace $S_{s}$ of $\mathrm{PG}(2 s-1, F)$ meets all $S^{I} \in \mathcal{S}_{s-1,1, F}^{I}$ only in points, then such points span $S_{s}$.

In [4] the previous result is seemingly proved using methods valid in any field with enough elements. However such a generalisation would contradict Theorem 3.3. In the opinion of the authors the proof in [4] is obtained using an erroneous argument. As a matter of fact, it is claimed in the proof at page 174 that the assumption $\langle\Phi\rangle=S_{s}$ is not used. However the contradiction $S_{s} \subset\left\langle\mathcal{S}_{s-2,1, \mathbb{C}}\right\rangle$ is inferred from $\Phi \subset \mathcal{S}_{s-2,1, \mathbb{C}}$.

A further counterexample, which exists whenever a hyperbolic quadric $Q^{+}(3, F)$ in a three-dimensional projective space admits an external line (a condition which is not met 
when the field $F$ is algebraically closed) is the following. If $\ell$ is the line corresponding to the two-dimensional vector space $\left\langle e_{1}\right\rangle \otimes\left\langle e_{1}^{\prime}, e_{2}^{\prime}\right\rangle$ and $m$ is a line external to the hyperbolic quadric obtained by the intersection of the Segre variety $\mathcal{S}_{2,1, F}$ with the 3 -space corresponding to the vector space $\left\langle e_{2}, e_{3}\right\rangle \otimes\left\langle e_{1}^{\prime}, e_{2}^{\prime}\right\rangle$, then the 3 -dimensional subspace $\langle\ell, m\rangle$ intersects $\mathcal{S}_{2,1, F}$ in the line $\ell$ belonging to $\mathcal{S}_{2,1, F}^{I I}$.

For the sake of completeness, a proof for corollary 16 is given.

Proof of corollary 16. Define

$$
S_{t}=\left\langle S_{s} \cap \mathcal{S}_{s-1,1, F}\right\rangle, t=\operatorname{dim} S_{t}
$$

and suppose $t<s$. It is proved in [4, p.173 (6)] that $S_{t} \subset\left\langle\mathcal{S}_{t-1,1, F}\right\rangle$ for some $\mathcal{S}_{t-1,1, F} \subset$ $\mathcal{S}_{s-1,1, F}$.

Note that $S_{s} \cap\left\langle\mathcal{S}_{t-1,1, F}\right\rangle=S_{t}$; otherwise, comparing dimensions, $S_{s}$ would intersect each $S^{I} \in \mathcal{S}_{t-1,1, F}$ in more than one point. Now choose

- a subspace $S_{s-t-1} \subset S_{s}$ such that $S_{s-t-1} \cap\left\langle\mathcal{S}_{t-1,1, F}\right\rangle=\emptyset$;

- a Segre variety $\mathcal{S}_{s-t-1,1, F} \subset \mathcal{S}_{s-1,1, F}$, such that $\left\langle\mathcal{S}_{s-t-1,1, F}\right\rangle \cap\left\langle\mathcal{S}_{t-1,1, F}\right\rangle=\emptyset$;

- two distinct $A^{I}, B^{I} \in \mathcal{S}_{s-t-1,1, F}^{I}$.

Since $\left\langle\mathcal{S}_{s-t-1,1, F}\right\rangle$ and $\left\langle\mathcal{S}_{t-1,1, F}\right\rangle$ are complementary subspaces of $\left\langle\mathcal{S}_{s-1,1, F}\right\rangle$, a projection map

$$
\pi:\left\langle\mathcal{S}_{s-1,1, F}\right\rangle \backslash\left\langle\mathcal{S}_{t-1,1, F}\right\rangle \rightarrow\left\langle\mathcal{S}_{s-t-1,1, F}\right\rangle
$$

is defined by $\pi(P)=\left\langle P \cup \mathcal{S}_{t-1,1, F}\right\rangle \cap\left\langle\mathcal{S}_{s-t-1,1, F}\right\rangle$.

Now suppose $\pi\left(S_{s-t-1}\right) \cap \mathcal{S}_{s-t-1,1, F}=\emptyset$. In $\left\langle\mathcal{S}_{s-t-1,1, F}\right\rangle$ consider

- the regulus $\mathcal{R}$ corresponding to $\mathcal{S}_{s-t-1,1, F}^{I}$, and the projectivity $\kappa: A^{I} \rightarrow B^{I}$ such that, for any $P \in A^{I}$, the line $\langle P, \kappa(P)\rangle$ belongs to $\mathcal{S}_{s-t-1,1, F}^{I I}$;

- the regulus $\mathcal{R}^{\prime}$ containing $A^{I}, B^{I}$ and $\pi\left(S_{s-t-1}\right)$, and the projectivity $\kappa^{\prime}: A^{I} \rightarrow B^{I}$ such that, for any $P \in A^{I}$, the line $\left\langle P, \kappa^{\prime}(P)\right\rangle$ is a transversal line of $\mathcal{R}^{\prime}$.

Since $F$ is an algebraically closed field, $\kappa^{\prime-1} \circ \kappa$ has a fixed point $P$. Therefore $\kappa(P)=$ $\kappa^{\prime}(P)$, so $\mathcal{R}$ and $\mathcal{R}^{\prime}$ have a common transversal. This contradicts $\pi\left(S_{s-t-1}\right) \cap \mathcal{S}_{s-t-1,1, F}=\emptyset$. So, a point $P \in S_{s-t-1}$ exists such that $\pi(P) \in \mathcal{S}_{s-t-1,1, F}$.

Next, let $C^{I} \in \mathcal{S}_{s-1,1, F}^{I}$ be such that $\pi(P) \in C^{I}$, and $Q$ the point in $\left\langle\mathcal{S}_{t-1,1, F}\right\rangle$ such that $Q, P$, and $\pi(P)$ are collinear. If $Q \in S_{t}$, then $\pi(P) \in S_{s}$, a contradiction; also $Q \in C^{I}$ leads to a contradiction (since it implies $P \in C^{I}$ ). So $Q \notin S_{t} \cup C^{I}$ and by a dimension argument two points $Q_{1} \in C^{I} \backslash S_{t}$ and $Q_{2} \in S_{t} \backslash C^{I}$ exist such that $Q, Q_{1}$ and $Q_{2}$ are collinear: they are on the unique line through $Q$ meeting both $C^{I} \cap\left\langle\mathcal{S}_{t-1,1, F}\right\rangle$ and a $(t-1)$ subspace of $S_{t}$ disjoint from $C^{I}$.

The plane $\left\langle P, Q_{1}, Q_{2}\right\rangle$ contains the lines $P Q_{2} \subset S_{s}$ and $\pi(P) Q_{1} \subset \mathcal{S}_{s-1,1, F}$ which meet outside $\left\langle\mathcal{S}_{t-1,1, F}\right\rangle$. This is again a contradiction. 